\title{
Diagnosis of HV Components by Digital PD Analyzer
}

\author{
Edward Gulski \\ Delft University of Technology, \\ High Voltage Laboratory, Delft, The Netherlands
}

\begin{abstract}
This paper reports about the development of a fingerprint technique to evaluate partial discharge (PD) measurements on $\mathrm{HV}$ components. Based on routine measurements in several actual $H V$ objects, the recognition of different $P D$ faults is discussed. The technique may help users identifying the cause of different types of insulation problems in HV equipment. More experience is still required to evaluate the effectiveness of this system on other independent samples.
\end{abstract}

\section{INTRODUCTION}

$\mathrm{H}^{\mathrm{s}}$ $\mathrm{V}$ equipment usually is tested for discharges using conventional discharge detection (bandwidths $\sim 300$ $\mathrm{kHz}$ ). Due to the increasing automation of PD measurements in recent years, the use of digital evaluation has become very popular [1-6]. It is known that the digital recording of measured information permits more systematic analysis of the discharge process.

In this paper practical implications of a fingerprint technique as introduced in the past [1], and as used in a recently commercialized $\mathrm{PD}$ analyzer (TEAS) $[4,7,8]$, will be presented here and will be discussed in the scope of the following goals.

The main goal of the automation is to create additional information about the source of the discharge. As a result, the electrical engineer will be aided in his decision about the quality and the conditions of an insulating system. In particular, the manufacturer of $\mathrm{HV}$ systems is interested in controlling the quality of his product, the testing institution is interested to certify the product, and finally the user of $\mathrm{HV}$ system is interested to know the residual life of his equipment.

Thus, there is a need to develop techniques to analyze the discharges and to produce a PD data bank for such purposes as development, manufacturing and monitoring of HV systems.

When the information of a discharge pattern can be quantified, resulting in a fingerprint of the measurement, it can be used for comparison with unknown situations [9]. In this way, most of specific design or manufacturing problems which may lead to discharge could be classified. As a result, a more efficient identification of discharge sources would be possible.

According to recent results, using continuous registration of PD over time, the degradation of the insulation at the discharge site may also be assessed $[10,12]$. 


\section{PD PATTERN RECOGNITION}

In the past a strong relationship has been found between the shape of $\mathrm{PD}$ patterns which occur in the 50 or $60 \mathrm{~Hz}$ sine wave and the type of defect causing them [11]. It is known that each discharge source with its geometry, location in insulation, dielectric properties and applied field is characterized by a specific sequence of discharges. An analysis of these sequences is thus a good means of discriminating between different discharge sources. Therefore, using digital processing, the PD pulses are grouped with respect to their intensity and their phase angle.

In this Section a concrete solution of a computer-aided PD detector will briefly be presented. A more detailed description of the system is given in $[4,5]$. The PD measuring circuit as used in these studies was composed of a coupling capacitor, a measuring impedance, and a $\mathrm{PD}$ detector, bandwidth 40 to $400 \mathrm{kHz}$ to measure and to display the PD pulses. In addition to this conventional PD detector, a digital instrument (TEAS) was used. It is known that the analog instrument which provides quasiintegration of the discharge current pulses has a pulse resolution time between two consecutive discharge pulses which is inversely proportional to the bandwidth of the measuring circuit. Moreover, to guarantee that no single discharge pulses as measured by the analog instrument is lost by the digital acquisition, the resolution time of the digital part has to be adjusted (during PD circuit calibration) to the pulse resolution of the analog system [8]. As a result, depending on the measuring circuit as used in particular cases, the time resolution of two consecutive discharge pulse was varied between 6 and $10 \mu \mathrm{s}$.

Using computer-aided processing, the PD pulses were grouped with respect to their phase angle. For this purpose, during the data acquisition the whole voltage cycle was divided into several phase windows, together representing the phase angle axis 0 to $360^{\circ}$. Due to the fact the resolution time of the data acquisition was varied between 6 and $10 \mu \mathrm{s}$, in case of $50 \mathrm{~Hz}$ power frequency 3333 to 2000 phase windows were used.

If the PD pulses are collected over several voltage cycles, in these phase windows the distribution of discharges can be observed. Moreover, based on these distributions three quantities can be determined: the number of $P D$ pulses, the maximum value of $P D$ magnitudes and the average value of $P D$ magnitudes.

These quantities, observed throughout the whole angle axis 0 to $360^{\circ}$, result in the following three distributions for the positive $(+)$ and the negative $(-)$ half of the voltage cycle [7]: the pulse count $H_{n} \pm(\phi)$, the mean pulse height $H_{q n} \pm(\phi)$ and the maximum pulse height $H_{q m a x} \pm(\phi)$. To provide the analysis of all these distributions and above all to quantify their characteristic shapes, the following statistical operators were introduced in the past $[4,7,12]$.

The asymmetry of $H_{q n}(\phi), H_{n}(\phi)$ and $H_{q \max }(\phi)$ is the quotient of the mean level in the $(+)$ and in the $(-)$ sets. The phase factor of $H_{g n}(\phi)$ is used to study the difference in inception voltage in the $(+)$ and the $(-)$ sets. The cross-correlation factor of $H_{q n}(\phi), H_{n}(\phi)$ and $H_{q \max }(\phi)$ evaluates the difference in shape between the $(+)$ and the $(-)$ sets. The number of peaks of $H_{q n} \pm(\phi), H_{n} \pm(\phi)$ and $H_{q \max } \pm(\phi)$ distinguishes between a distribution with one single peak and a distribution with several peaks. The skewness of $H_{q n} \pm(\phi), H_{n} \pm(\phi), H_{q \max } \pm(\phi)$, as an indicator for the asymmetry of a distribution with respect to a normal distribution. The kurtosis of $H_{q_{n}} \pm(\phi)$, $H_{n} \pm(\phi), H_{g \max } \pm(\phi)$ is an indicator of the deviation from the normal distribution.

After a PD measurement has been finished, the above mentioned statistical operators are processed [8]. In Figure 4 an example of phase-resolved distributions and their fingerprint are shown. The time duration which is needed to collect the data depends on the intensity of the PD process itself. The experiments as discussed in this paper have shown, that in practice between 2 and $10 \mathrm{~min}$ are sufficient to produce the characteristic fingerprint of a $\mathrm{PD}$ source.

Using the fingerprint of a particular measurement, a comparison with fingerprints of normalized PD sources like cavity discharges, surface discharges, corona discharges etc. is possible. For this purpose, using mathematical pattern recognition methods, the percentage can be obtained which reflects the recognition of a particular measurement as a defined discharge type in the reference data [9]. In Figure 4 an example of such classification is shown. More detailed description of this technique, called centour score, is given in [9]. In particular, for the number of statistical operators of reference data a collection of fingerprints occurs. The mathematical center $C$ of these fingerprints is determined and the position of an unknown discharge as represented by a set of statistical operators $X$ can be compared with this center. The recognition score is now defined as the percentile rank of the data (fingerprints) which are further away from the center $C$ of the known discharge population than the measured value of an unknown discharge $X$. This percentage is not the same as the probability that $X$ is indeed identical to the standard defect, but it gives a reasonable reflection of it. The score of the unknown 
defect is $80 \%$ if eighty percent of the fingerprints of the reference data are further away from $C$ than $X$ itself.

\section{PD DATA BANK}

When several fingerprints are available, it is possible to develop a collection containing specific data: the PD data bank. In this way an unknown discharge measurement as represented by its fingerprint can be compared to a collection of known situations.

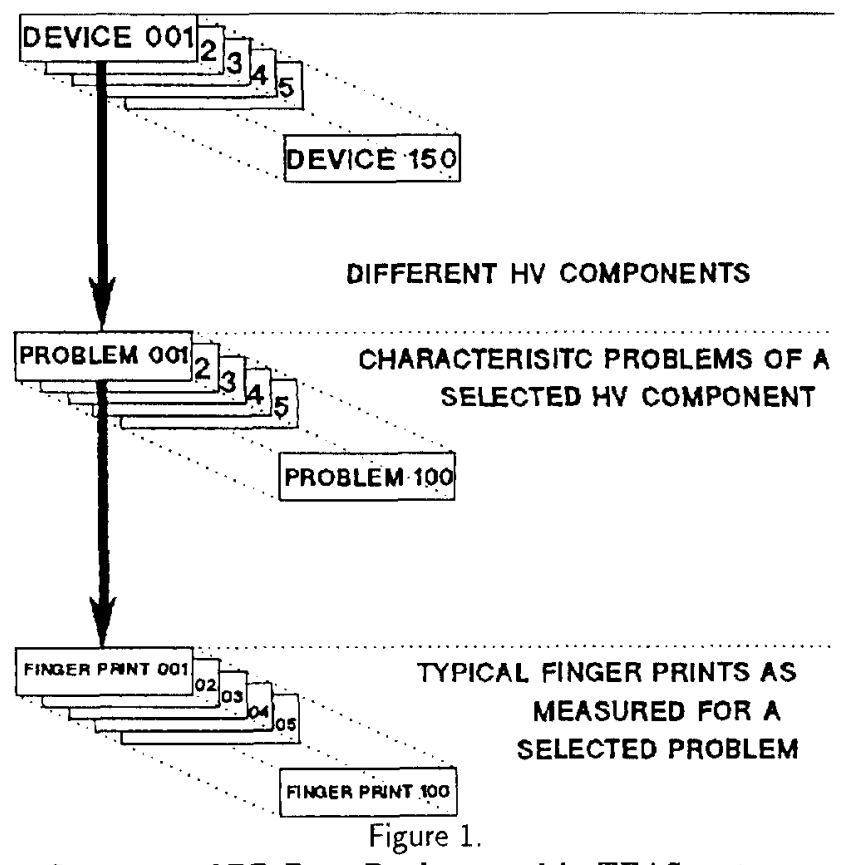

Structure of PD Data Bank as used in TEAS system.

A data bank is judged to be well designed if it produces a high similarity for the correct defect and low or nil for all the others. If no recognition is possible, the result should be low or of nil similarity for all defects.

Of course, the result of such a recognition process strongly depends on the following factors: the test conditions at which the reference data are obtained, the number of measurements used to represent a defect and the way the data bank is organized.

From a practical point of view it is attractive to organize such a data bank using the following structure with two levels of storage, see Figure 1.

At the device level, here each device represents one of different groups of PD problems, for instance 'Artificial defects', 'Epoxy insulated current transformer', 'Paper/oil bushing', 'GIS', etc.

At the problem level, each of the above mentioned devices is characterized by means of specific problems, for instance the device 'Artificial defects' contains cavity discharges, surface discharges, corona discharges etc. An other device for instance 'GIS' may contain problems which may occur in the case of GIS system only.

In the next Section a list of 17 problems representing the device 'Artificial defects' is shown. An example of a device where industrial problems of a $\mathrm{HV}$ system are defined is discussed in Section 5. Using practical examples, the application of the above mentioned technique will be shown using two kinds of PD data bank.

\section{RECOGNITION USING 'ARTIFICIAL DEFECTS'}

The device called 'Artificial defects' contains 17 problems corresponding to simple two-electrode models, representing 17 possible defects in the insulation. In the following PD sources, their model description as well as the test conditions are listed which were used for analysis of discharges in $\mathrm{HV}$ components.

1. Single corona discharges at the HV electrode in air. Diameter of $\mathrm{HV}$ point $100 \mu \mathrm{m}$; distance to $\mathrm{LV}$ electrode $30 \mathrm{~mm}$; test voltage $2.2 \mathrm{kV}_{\text {eff }}$.

2. Single corona discharges at the $\mathrm{LV}$ electrode in air. Diameter of the low voltage point $100 \mu \mathrm{m}$; distance to $\mathrm{HV}$ electrode $30 \mathrm{~mm}$; test voltage $2.3 \mathrm{kV}_{\text {eff }}$.

3. Surface PD between external LV electrode and PE surface. LV cylinder-to-PE plane system. Dielectric: polyethylene. LV electrode diameter $20 \mathrm{~mm}$; average field strength $2.8 \mathrm{kV} / \mathrm{mm}$.

4. Surface PD between external HV electrode and PE surface. HV cylinder-to-PE-plane system. Dielectric: polyethylene. $\mathrm{HV}$ electrode diameter $20 \mathrm{~mm}$; average field strength $3.4 \mathrm{kV} / \mathrm{mm}$.

5. Multiple corona discharges at the HV electrode in air. Diameter of HV points 50 to $200 \mu \mathrm{m}$. Distance to $\mathrm{LV}$ electrode $25 \mathrm{~mm}$; test voltage $16 \mathrm{kV}_{\text {eff }}$.

6. Multiple corona discharges at the LV electrode in air. Diameter of low voltage points 50 to $200 \mu \mathrm{m}$. Distance to $\mathrm{HV}$ electrode $15 \mathrm{~mm}$; test voltage $5.6 \mathrm{kV}_{\text {eff }}$.

7. Surface discharges between two dielectric surface. Test voltage $=29 \mathrm{kV}_{\text {eff }}$.

8. Contact noise. Imperfect metal to metal joint in the $\mathrm{HV}$ electrode. Test voltage $2.2 \mathrm{kV}_{\text {eff }}$.

9. Floating object. Badly grounded metallic component near the $\mathrm{HV}$ circuit. Ungrounded metallic plate at 500 $\mathrm{mm}$ distance from the $\mathrm{HV}$ terminal of a discharge free test object; test voltage $28 \mathrm{kV}_{\text {eff }}$.

10. PD on external dielectric. Two touching insulated conductors; surfaces PD between two touching 6/10 $\mathrm{kV}$ PE cables; test voltage $13 \mathrm{kV}_{\text {eff } f}$. 
11. LV Electrode-bounded cavity. Dielectric: polyethylene; Flat cavity between $\mathrm{PE} /$ metal $16 \times 0.4 \mathrm{~mm}^{2}$. Test voltage $11 \mathrm{kV}_{e f f}$; average field strength $2.43 \mathrm{kV} / \mathrm{mm}$.

12. Dielectric-bounded flat cavity. Dielectric: polyethylene; Flat cavity in PE $16 \times 0.4 \mathrm{~mm}^{2}$. Test voltage 11 $\mathrm{kV}_{\text {eff }} ;$ average field strength $2.35 \mathrm{kV} / \mathrm{mm}$.

13. Dielectric-bounded cavities. Dielectric: polyethylene; Flat cavities in PE; 5 cavities of $4 \times 0.4 \mathrm{~mm}^{2}$. Test voltage $12 \mathrm{kV}_{\text {eff }}$; average field strength $2.7 \mathrm{kV} / \mathrm{mm}$.

14. LV Electrode-bounded cavities. Dielectric: polyethylene; Flat cavities between $\mathrm{PE} /$ metal; 5 cavities of $4 \times 0.4 \mathrm{~mm}^{2}$. Test voltage $12 \mathrm{kV}_{\text {eff }}$; average field strength $2.9 \mathrm{kV} / \mathrm{mm}$.

15. Fissure PD between PE films. Fissure between two PE layers HV electrode: $0.05 \mathrm{~mm}$ aluminum foil. PD due to high tangential field strength in the fissure at the end of HV electrode; PD occur between two 0.1 mm PE foils. Test voltage $=12 \mathrm{kV}_{e f f}$.

16. Background noise.

17. No partial discharges.

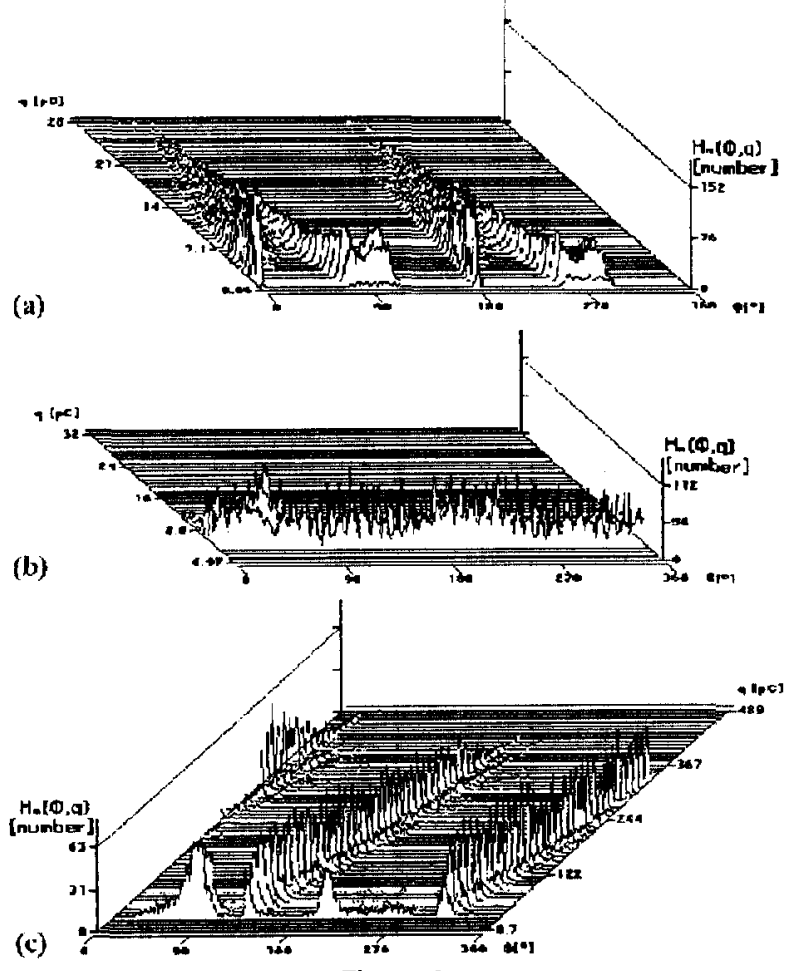

Figure 2.

The 3D plots as observed on different $\mathrm{HV}$ components.

Each of these defects is represented by series of 279 fingerprints as obtained for one and the same type defect (between 5 and 9 different samples) and stored in the device 'Artificial defects', each as a separate problem. In the following, this collection of known fingerprints will be used to recognize unknown discharges in HV systems. In Figure 2 three examples of a 3 dimensional relationship between discharge magnitude, $\mathrm{PD}$ in- tensity and the phase angle $H_{n}(\phi, q)$ distribution are presented. As shown, different insulation defects in industrial objects are characterized by typical differences in the landscape of such 3-dimensional figures. It confirms the opinion that these $H_{n}(\phi, q)$ distributions as mentioned here might be very useful to analyze the discharge processes in actual $\mathrm{HV}$ equipment $[1,10]$.

In the following this fingerprint collection of artificial defects will be applied to recognize PD sources in three HV components: $23 \mathrm{kV}$ insulator, $10 \mathrm{nF}$ capacitor and $245 \mathrm{kV}$ instrument transformer.

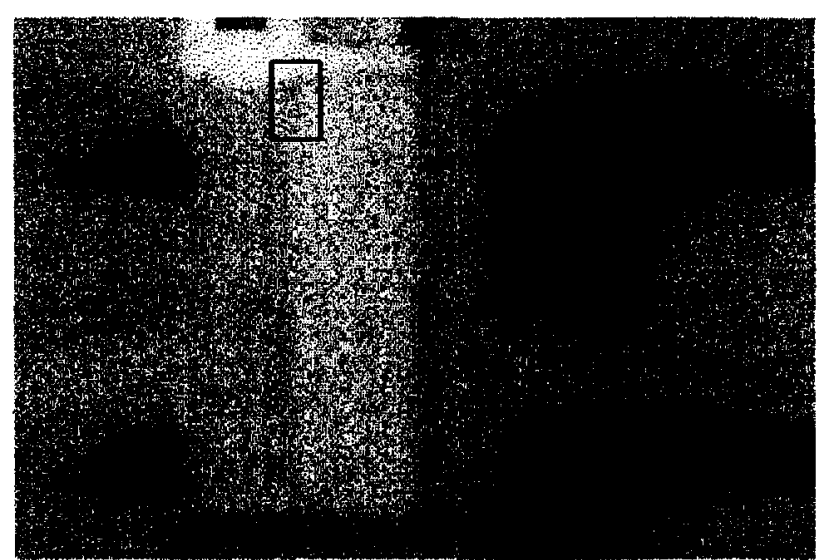

Figure 3 .

Photograph of the cross section of the $23 \mathrm{kV}$ epoxy insulator showing the location of air pockets.

\subsection{CAVITY DISCHARGES IN EPOXY RESIN INSULATOR}

In a $23 \mathrm{kV}$ insulator the origin of discharges was related to air pockets around the ceramic core of the insulator which remained after casting, see Figure 3 . The evidence of these defects was found by X-ray photographs.

In Figure 4 the phase-position quantities $H_{q \max }(\phi)$, $H_{q_{n}}(\phi)$ and $H_{n}(\phi)$ as obtained during 2 min at $23 \mathrm{kV}$ are shown. Furthermore, in this Figure the fingerprint and the result of recognition are shown. In Figure 2 the $3 \mathrm{D}$ plot of the $H_{n}(\phi, q)$ distribution as processed during this test is shown.

The comparison to the 'Artificial defects' recognizes clearly internal discharges ( $98 \%$ ) cavities dielectric bounded; the resemblance to other defects is low. 


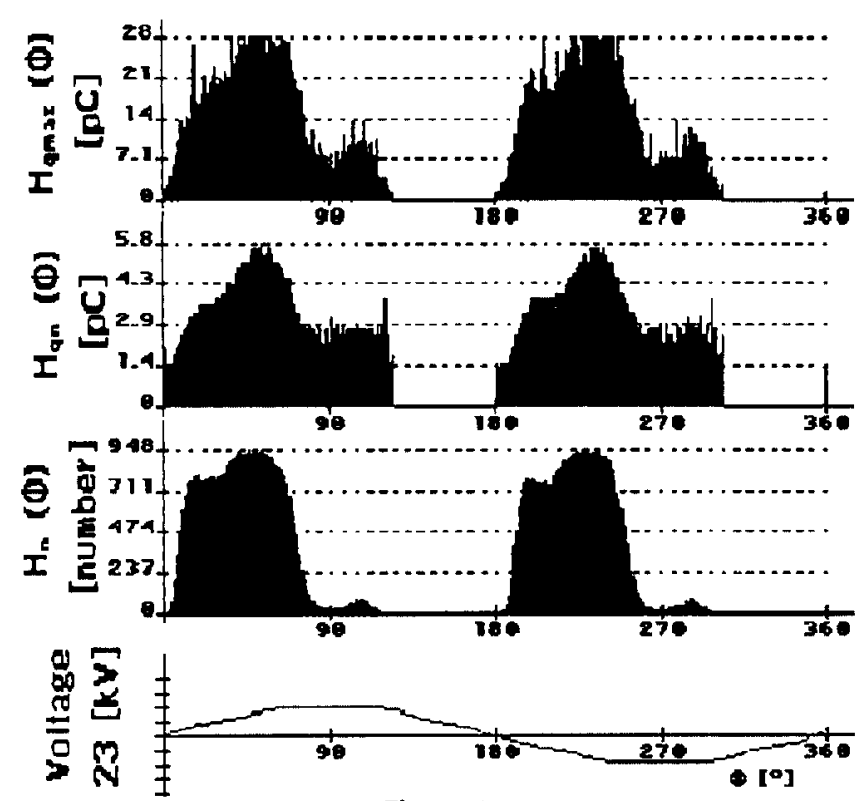

Figure 4.

The phase-position distributions, the fingerprint, and the recognition by data bank Artificial defect as observed on $23 \mathrm{kV}$ epoxy insulator. The origin of $\mathrm{PD}$ reveals air pockets around ceramic core.

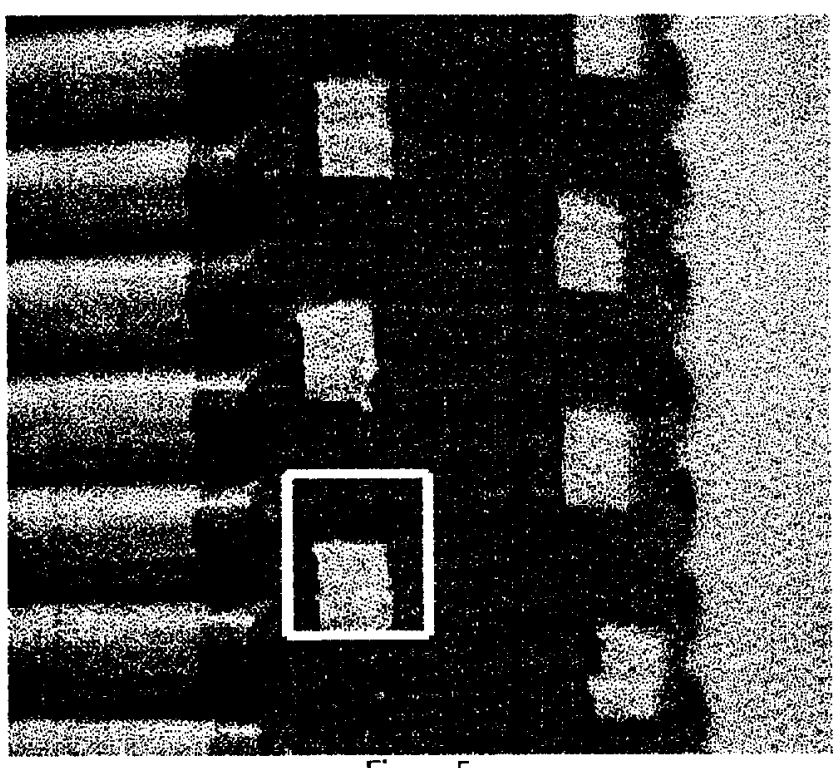

Figure 5.

Photograph of capacitor packages showing a solder joint.

\subsection{CONTACT NOISE DISCHARGE IN MIXED INSULATION CAPACITOR}

A $10 \mathrm{nF} / 200 \mathrm{kV}$ capacitor showed discharges which are due to a bad solder joint between capacitor packages inside the capacitor, see Figure 5. The evidence of this defect was found by visual examination after dismantling the test object.

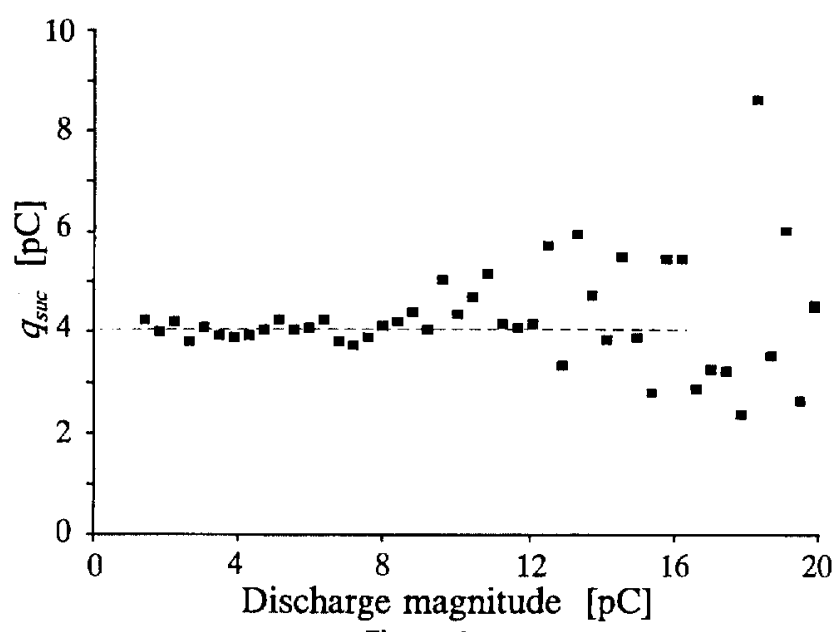

Figure 6.

The phase-position distributions, the fingerprint, and the recognition by data bank 'Artificial defects' as observed on $10 \mathrm{nF} / 220 \mathrm{kV}$ mixed insulation capacitor. The origin of $P D$ reveals a bad solder joint between capacitor packages inside the capacitor.

The object was tested during 2 min at $160 \mathrm{kV}$ test voltage. In Figure 6 the phase position quantities $H_{q \max }(\phi)$, $H_{q n}(\phi)$ and $H_{n}(\phi)$ as well as the fingerprint and the result of comparison to 'Artificial defects' are shown. In Figure 2 the $3 \mathrm{D}$ plot of the $H_{n}(\phi, q)$ distribution as processed during this test is shown.

It follows from Figure 6 that the contact noise (72\%) was clearly recognized and the floating part (38\%) to a lesser extent. Also there is no resemblance to other defects.

\subsection{FLOATING PARTICLE DISCHARGES IN CT/PT COMBINATION}

A $245 \mathrm{kV}$ paper/oil combination of a current transformer/potential transformer (CT/PT) has shown discharges which are caused by a floating aluminum screen inside the transformer. The evidence of this defect was found by visual examination after dismantling the test object. This transformer was tested during 2 min at 395 $\mathrm{kV}$. In Figure 7 the phase position quantities $H_{\text {qmax }}(\phi)$, $H_{q n}(\phi)$ and $H_{n}(\phi)$ as well as the fingerprint and the result of comparison to 'Artificial defects' are shown. In Figure 2 the 3D plot of the $H_{n}(\phi, q)$ distribution as processed during this test is shown. The classification diagram confirms the origin of discharges: $94 \%$ floating part. There is no resemblance to other defects. 


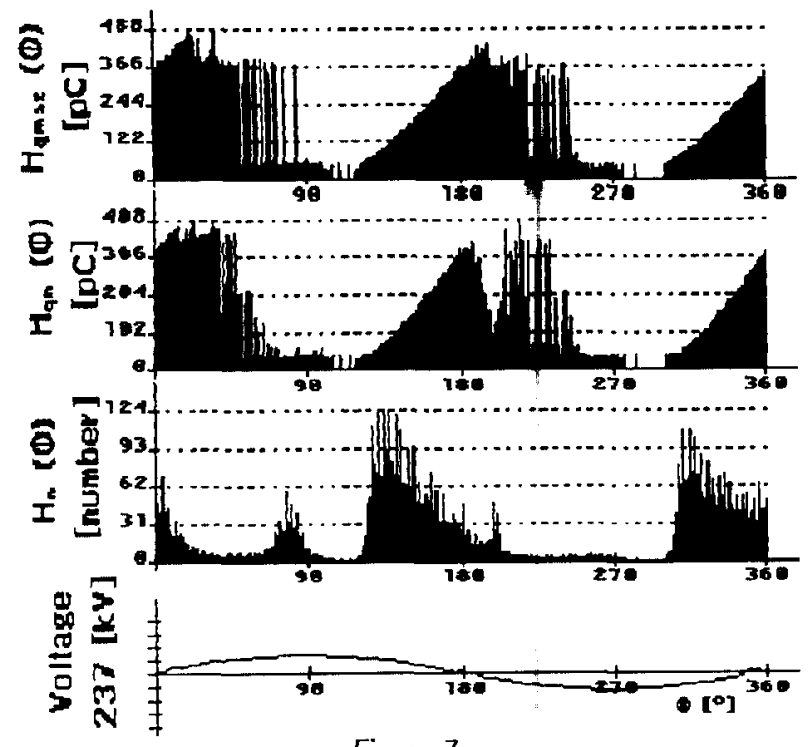

Figure 7 .

The phase-position distributions, the fingerprint, and the recognition by data bank 'Artificial defects' as observed on $245 \mathrm{kV}$ CT/PT combination. The origin of $\mathrm{PD}$ reveals floating aluminum screen inside the transformer.

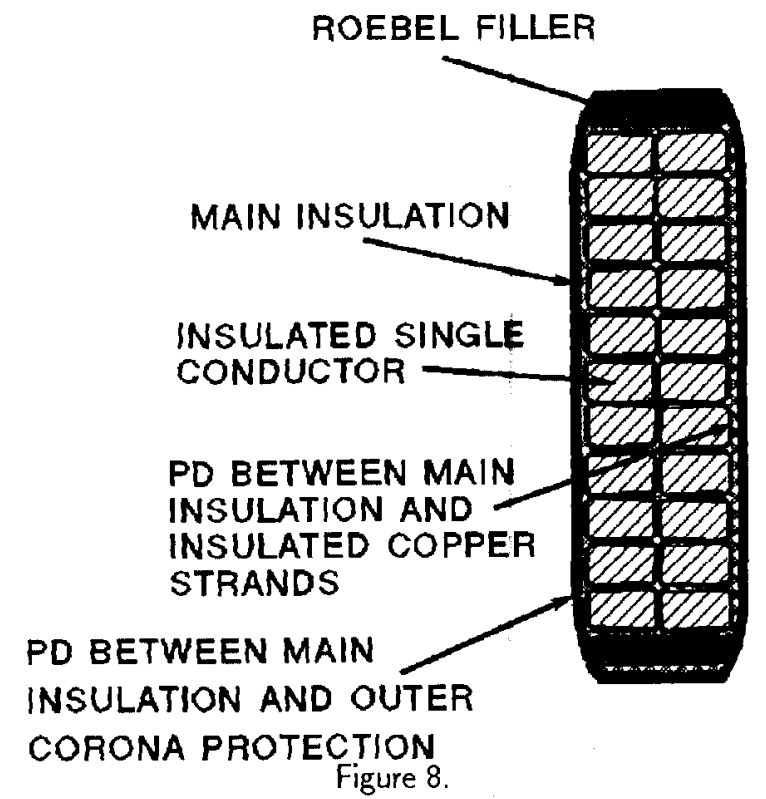

(a) schematic cross section of a stator bar showing location of studied PD defects, (b) photograph of the stator test sample.

\section{RECOGNITION USING INDUSTRIAL CASES}

As shown in the previous Section using physical models of different PD sources, their fingerprints can be used to recognize defects in actual industrial objects. Experience has shown that the recognition of industrial defects
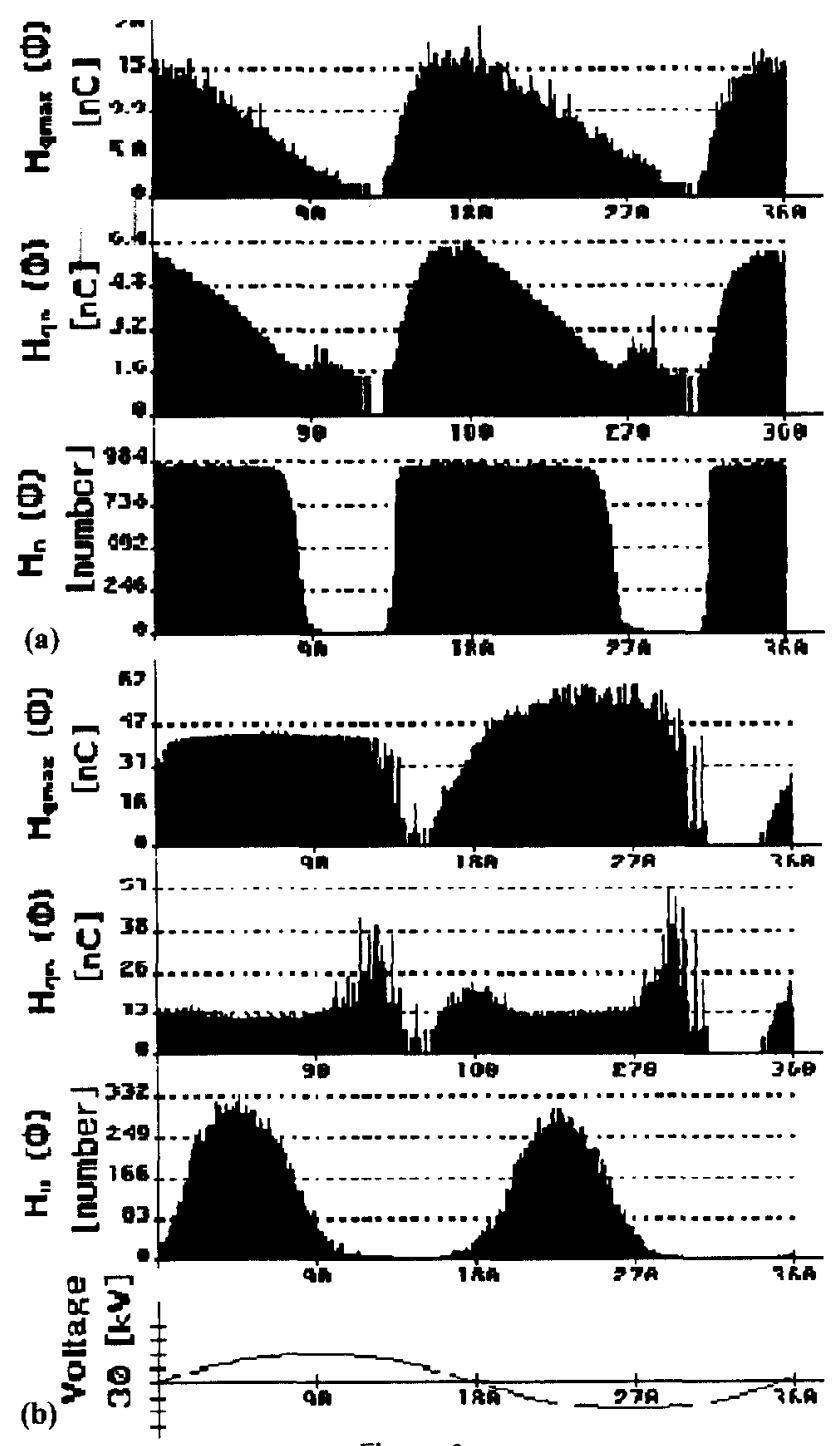

Figure 9.

Phase-resolved distributions and the recognition by data bank 'Artificial defects' as observed for different defects in two 306 MVA turbogenerator bars. (a) PD in gaps between main insulation and single conductors insulation, (b) PD between single conductors insulation.

depends on how near the artificial defects come to actual cases. In Figures 8 and 9 two examples are shown where recognition using data bank 'Artificial defects' is used to recognize a known defect in the insulation of two generator stator bars: PD in gaps between main insulation and insulated copper strands, and PD between strands. It follows from Figure 9 that the use of artificial defects also has its limitations. Moreover, factors like roughness of the interface surface, the shape of the defect, the aging process, local field strength etc. of a particular defect in a real component may influence the measured PD sequence which is the basis for the recognition. A systematic study 
of this relationship was out of the scope of this paper.

It is known also that with regard to a particular $\mathrm{HV}$ system a number of discharges can occur, which are only specific for this component, and their modeling does not make much sense.

Therefore it is equally important to develop a second type of PD data bank which shall contain HV systems and industrial problems.

With the fingerprints of these discharges, a catalog of possible 'Problems' can be made. Such a collection is of importance when in the future similar situations occur.

In the following practical examples are presented of a device called 'Reactor coils $350 / 400 \mathrm{kV}$ '. This device was developed after the analysis of discharges in different reactor coils of 75,350 and $400 \mathrm{kV}$ transformer test sets. Moreover, all defects as mentioned in this Section could actually be found by visual inspection and could be repaired.

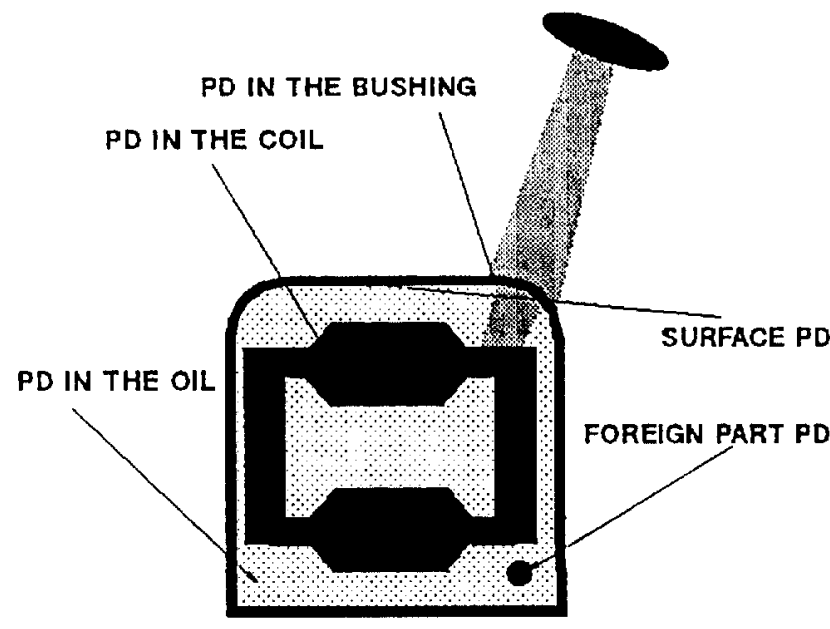

Figure 10 .

Schematic cross section of a transformer test set showing location of industrial defects.

In particular five different discharge sources were studied and their fingerprints were used to define the industrial problems of test transformers, see Figure 10. Bushing discharges in $350 \mathrm{kV}$ reactor coil, coil discharges in 350 $\mathrm{kV}$ reactor coil, foreign part discharges in $400 \mathrm{kV}$ reactor coil, and surface corona discharges in $400 \mathrm{kV}$ reactor coil,

\section{OIL DISCHARGES IN $400 \mathrm{kV}$ REACTOR COIL,}

In Figures 11 and 12 examples of 3-dimensional distribution $H_{n}(\phi, q)$ as observed for the above mentioned

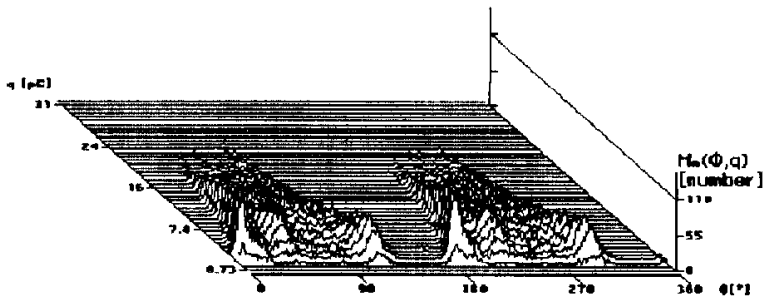

Figure 11.

The $3 \mathrm{D}$ plots as observed on different $\mathrm{HV}$ components.

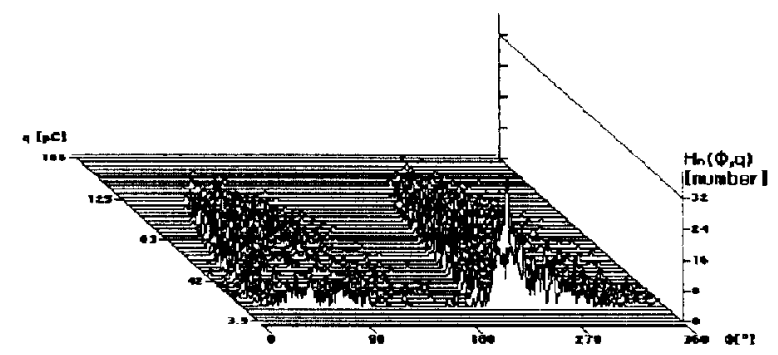

Figure 12.

The 3D plots as observed on different HV components.

industrial problems are shown. Similar to results in Figure 2 , the same conclusions can be drawn from these diagrams. First of all these five groups, each represented by at least 30 fingerprints, were mutually compared in order to see whether and in what measure they could be distinguished from each other.

This was done by calculating the classification if one of the five fingerprints is compared with either itself (self recognition) or with the other four. This proved to be quite successful: self recognition was 98 to $100 \%$, whereas the classification fell below $40 \%$ when the other defects were entered.

This proves that sufficient distinction between these five 'industrial problems' exists. In the following, the above mentioned five industrial problems are discussed where in total nine 350 and $400 \mathrm{kV}$ test transformers are tested. Due to the low number of test objects, the main goal of this comparison is the introduction of this analysis method and not systematic test of the performances of this method. Using only one sample of a $75 \mathrm{kV}$ reactor coil the effectiveness of this method to detect unknown insulation problem was possible.

\subsection{BUSHING DISCHARGES IN $350 \mathrm{kV}$ REACTOR COIL}

The $350 \mathrm{kV}$ transformer test set showed discharges caused by a bad connection of the bushing inside the 


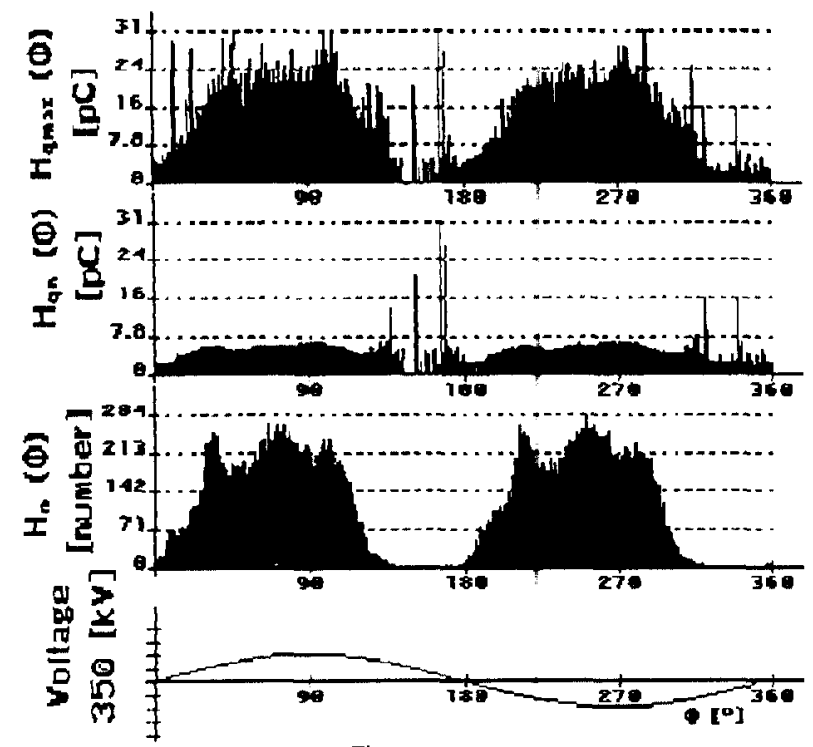

Figure 13 .

The phase-position distributions, the fingerprint, and the recognition by data bank 'Reactor coils: $350 / 400 \mathrm{kV}$ ' as observed on bushing discharges in $350 \mathrm{kV}$ reactor coil.

transformer tank. Four tests of $2 \mathrm{~min}$ were carried out at $350 \mathrm{kV}$ test voltage. The collection of 120 fingerprints was used to define the 'industrial problem': $350 \mathrm{kV} \mathrm{Re}-$ actor: PD in bushing

In Figure 13 examples of the phase-position quantities, their fingerprint and the results of the recognition are shown. In Figure 11 the $3 \mathrm{D}$ plot of the $H_{n}(\phi, q)$ distribution as processed during this test is shown. It follows from the classification that the type of defect 'PD in bushing' is clearly recognized $100 \%$. The resemblance to other defects as measured in both types of the transformer is low.

\subsection{COIL DISCHARGES IN $350 \mathrm{kV}$ REACTOR COIL}

Internal discharges were found inside a coil of a $350 \mathrm{kV}$ transformer test set. Nine tests of $5 \mathrm{~min}$ were performed at $200 \mathrm{kV}$ test voltage. The collection of 270 fingerprints were used to define the 'industrial problem': $350 \mathrm{kV}$ reactor: PD in coil.

In Figure 14 examples of the phase-position quantities, their fingerprint and the result of the recognition are shown. In Figure 11 the $3 D$ plot of the $H_{n}(\phi, q)$ distribution as processed during this test is shown. It follows from the classification diagram that this type of defect: 'PD in coil' is $100 \%$ recognized. There is no resemblance to other defects.

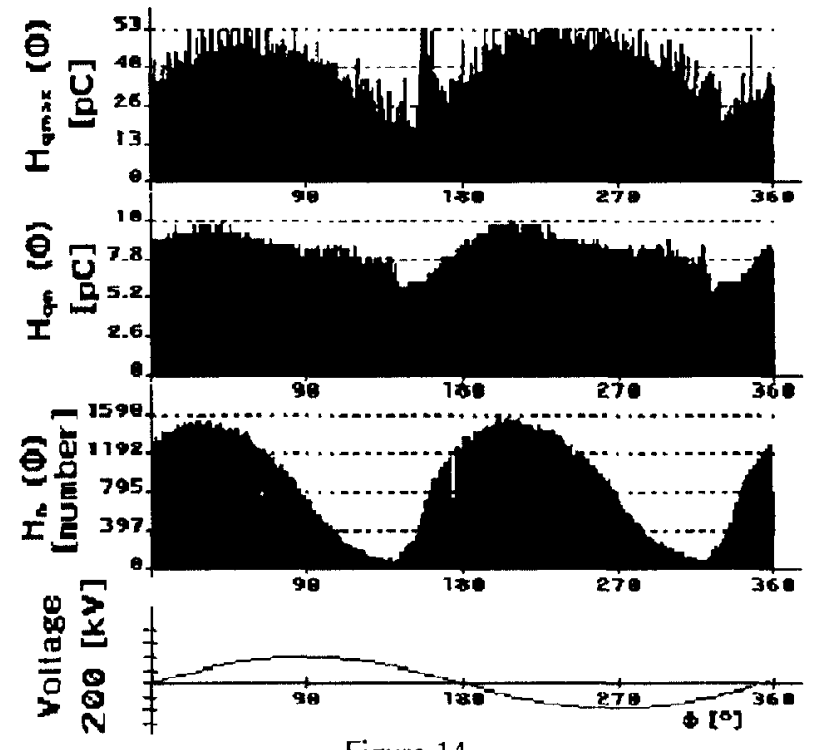

Figure 14

The phase-position distributions, the fingerprint, and the recognition by data bank 'Reactor coils: 350 to $400 \mathrm{kV}$ ' as observed on coil discharges in $350 \mathrm{kV}$ reactor coil.

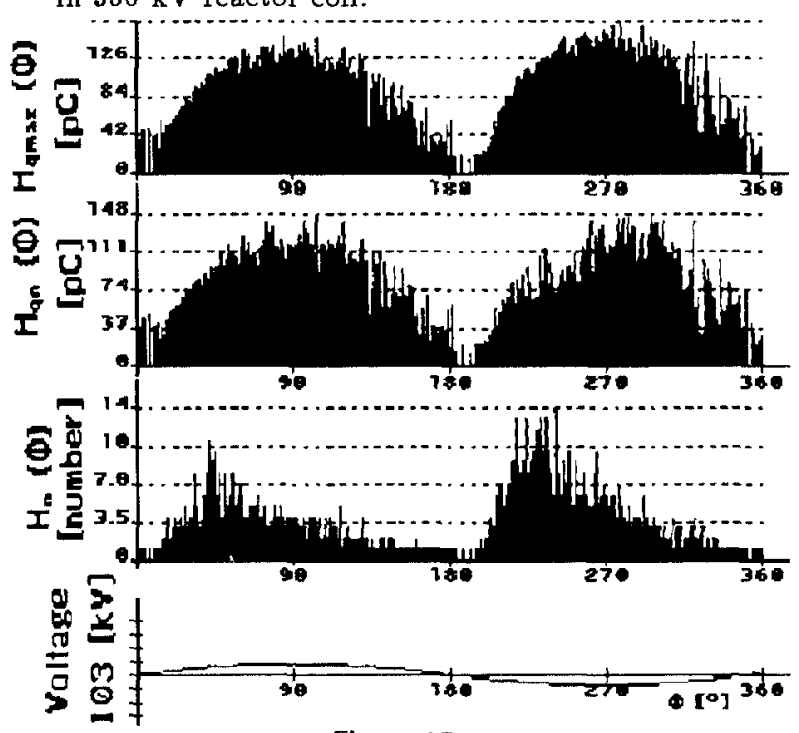

Figure 15.

The phase-position distributions, the fingerprint, and the recognition by data bank 'Reactor coils:350 to $400 \mathrm{kV}$ ' as observed for foreign part discharges in $400 \mathrm{kV}$ reactor coil.

\subsection{FOREIGN PART DISCHARGES IN $400 \mathrm{kV}$ REACTOR COIL}

This $400 \mathrm{kV}$ transformer test set showed discharges caused by small dielectric particles between the coil and the internal screen. Two tests of $2 \mathrm{~min}$ were carried out at $100 \mathrm{kV}$ test voltage. The collection of 60 fingerprints were used to define the 'industrial problem': $400 \mathrm{kV}$ reactor: PD/foreign part. In Figure 15 an example of the 
phase-position quantities, their fingerprint and the result of the recognition are shown. In Figure 12 the $3 \mathrm{D}$ plot of the $H_{n}(\phi, q)$ distribution as processed during this test is shown.

It follows from the classification that this type of defect:' PD/foreign part' can be $100 \%$ recognized. The resemblance to other defects as measured in both types of transformer is $<23 \%$.

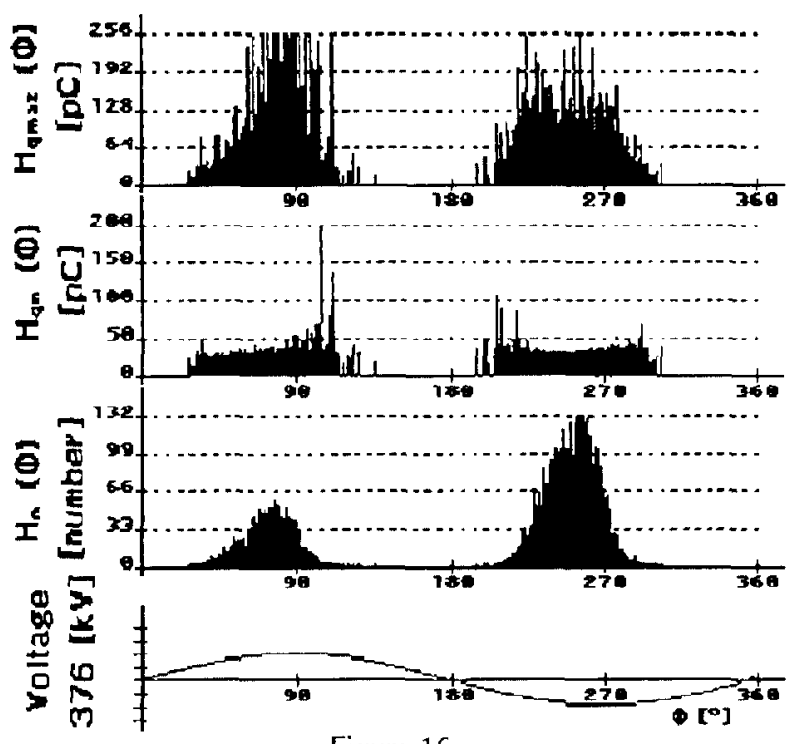

Figure 16.

The phase-position distributions, the fingerprint, and the recognition by data bank 'Reactor coils: 350 to $400 \mathrm{kV}$ ' as observed for surface discharges in $400 \mathrm{kV}$ reactor coil.

\subsection{SURFACE DISCHARGE IN $400 \mathrm{kV}$ REACTOR COIL}

Surface discharges were found on the cylinder surface of a $400 \mathrm{kV}$ transformer test set. Five tests of 2 min were performed at $376 \mathrm{kV}$ test voltage. The collection of 150 fingerprints were used to define the 'industrial problem': $400 \mathrm{kV}$ reactor: surface PD.

In Figure 16 an example the phase-position quantities, their fingerprint and the result of the recognition are shown. In Figure 12 the $3 \mathrm{D}$ plot of the $H_{n}(\phi, q)$ distribution as processed during this test is shown. It follows from the classification diagram that this type defect: 'surface $\mathrm{PD}$ ' is $100 \%$ recognized. There is no resemblance to other defects.

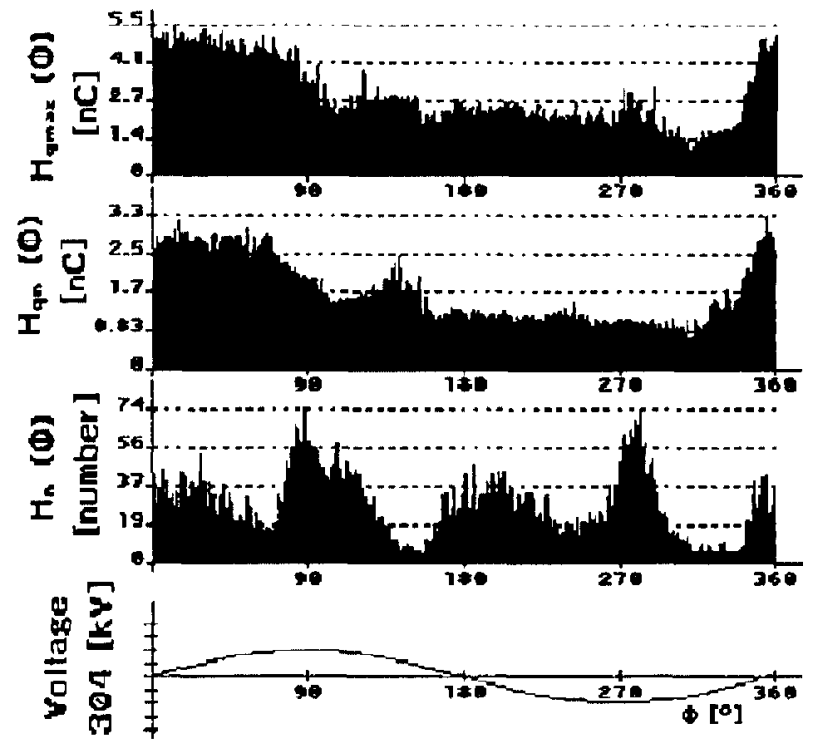

Figure 17.

The phase-position distributions, the fingerprint, and the recognition by data bank 'Reactor coils: 350 to $400 \mathrm{kV}$ ' as observed for oil discharges in $400 \mathrm{kV}$ reactor coil.

\subsection{OIL DISCHARGES IN $400 \mathrm{kV}$ REACTOR COIL}

Discharges caused due to high moisture content of the oil were found in this type of $400 \mathrm{kV}$ transformer test set. One test of $2 \mathrm{~min}$ was performed at $304 \mathrm{kV}$ test voltage. The collection of 30 fingerprints were used to define the 'industrial problem': $400 \mathrm{kV}$ reactor: PD in oil.

In Figure 17 an example the phase-position quantities, their fingerprint and the result of the recognition are shown. In Figure 12 the $3 \mathrm{D}$ plot of the $H_{n}(\phi, q)$ distribution as processed during this test is shown. It follows from the classification that this type defect: 'PD in oil' is $100 \%$ recognized. There is only a small resemblance $(27 \%)$ to other defects.

\subsection{COIL DISCHARGES IN $75 \mathrm{kV}$ REACTOR COIL}

This $75 \mathrm{kV}$ transformer test set showed discharges caused inside the coil. At a voltage of $72 \mathrm{kV}$ a 5 min test was made and the results were compared to the industrial problem' of reactor coils: $350 / 400 \mathrm{kV}$.

In Figure 18 the phase-position quantities, their fingerprint as well as the result of the recognition are shown. In Figure 11 the $3 \mathrm{D}$ plot of the $H_{n}(\phi, q)$ distribution as 


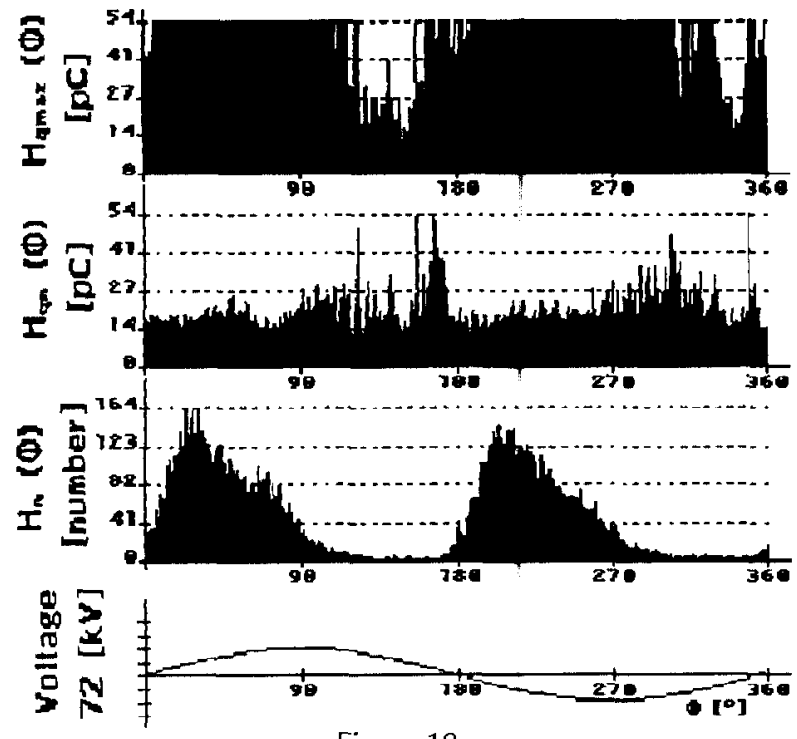

Figure 18.

The phase-position distributions, the fingerprint, and the recognition by data bank 'Reactor coils: 350 to $400 \mathrm{kV}^{\prime}$ as observed on coil discharges in $75 \mathrm{kV}$ reactor coil.

processed during this test is shown. Looking at the classification diagram, 95\% resemblance to the 'industrial problem': $350 \mathrm{kV}$ reactor: PD in coil is clearly visible.

Using this example (blind testing) the applicability of fingerprints of one type of defect, as obtained using different HV systems, is shown. In other words this example shows, that it's possible using collection of industrial defects to recognize an unknown defect in other type of HV component.

\section{CONCLUSIONS}

7 HIS presented attempt to apply the fingerprint techI nique to develop a $\mathrm{PD}$ data bank of full scale $\mathrm{HV}$ systems is encouraging. The results obtained are summarized as follows.

It has been shown that the use of conventional detection combined with statistical analysis techniques can be used for recognition of different discharge sources.

The proposed device/problem structure for the storage of fingerprints in a PD data bank is straightforward and can efficiently be used.

It is possible, using physical models of discharges (artificial defects) to prove their presence in complete $\mathrm{HV}$ systems. In this way the applicability of results as obtained from physical models of discharges for the evaluation of the full scale HV systems is possible. However this way of recognition of $\mathrm{PD}$ sources has also its limitation, see recognition of discharges as shown in Section 4.

It is possible, using the fingerprint techniques to classify the properties of several 'industrial defects' in specific types of HV equipment. Moreover, this information may be useful to recognized defects and to take the measures to make repairs, see the recognition of coil discharges in a $75 \mathrm{kV}$ test transformer using the collection of fingerprints as obtained for 350 and $400 \mathrm{kV}$ transformers.

Using the presented method the recognition of defects in actual industrial objects becomes more realistic. Needless to say, experience still has to be acquired with using the 'industrial defects' data base on $\mathrm{HV}$ equipment with unknown problems. This task will require many years of correlating fingerprints with actual conditions in the test departments of the manufacturers, the testing institutions and the users.

\section{REFERENCES}

[1] B. H. Ward, "Digital Techniques for PD Measurements", IEEE Panel Session, Digital Techniques in HV Tests, Long Beach, pp. 83-87, July 1989.

[2] J. Fuhr, M Haessig, B. Fruth, T. Kaiser, "PDfingerprints of some HV Apparatus", Proc. of IEEE Int. Symp. on EI, Toronto, pp. 129-132, June 3-6, 1990.

[3] R. Krump, Ein störresistentes Verfahren Zur computergestützten TE-diagnostik in $S_{6}$ Schaltanlagen, Thesis of Wuppertal University, 1989

[4] E. Gulski, "Computer-aided Measurement of PD in HV Equipment", IEEE Trans. on Elec. Insulation, Vol. 28, No. 6, pp. 969-983, 1993.

[5] Ch. Hantouche, D. Fortune, "Digital Measurement of PD in Full-sized Power Capacitors", IEEE Trans. on Elec. Insulation, Vol. 28, No. 6, pp. 1025-1032, 1993.

[6] T. R. Blackburn, R. E. James, B. T. Phung, S. L. Jones, "Advanced Techniques for Characterization of PD in Oil-impregnated and Gas Insulated Systems", Paper 15-102, CIGRE Session, Paris, 1994.

[7] E. Gulski, Computer-aided Recognition of PD Using Statistical Tools, Delft University Press, 1991

[8] E. Gulski, P. Seitz, "Computer-aided Registration and Analysis of uPd in HV Equipment", in Proceedings of 8th Intern. Symp. on HV Engineering, Yokohama Japan, 1993. 
[9] F. H. Kreuger, E. Gulski, A. Krivda, "Classification of PD", IEEE Trans. on Elec. Insulation, Vol. 28, No. 6, pp. 917-931, 1993.

[10] F. H. Kreuger, P. H. F Morshuis, E. Gulski, "Evaluation of Discharge Damage By Fast Transient Detection and Statistical Analysis", Paper 15-106, CIGRE Session, 1994, Paris.

[11] F. H. Kreuger, Discharge Detection in HV Equipment, London, A. Heywood Book, 1964.
[12] T. Tanaka, O. Okamoto, "Analysis of $q-n$ and $\phi-n$ Characteristics of PD in Several Electrode Systems", Proc. of IEEE of IEEE Int. Symp. on EI, pp. 190-193, June 5-8, 1978.

This paper is based on a presentation given at the 1994 Volta Colloquium on Partial Discharge Measurements, Como, Italy, 31 August - 2 September 1994.

Manuscript was received on 28 October 1994, in final form $3 \mathrm{July}$ 1995. 